\title{
Chasing the Curve: Program Description of the Geisinger Primary Care Behavioral Health Virtual First Response to COVID-19
}

\author{
Sean M. O’Dell ${ }^{1,2}$, Shelley J. Hosterman ${ }^{1}$, Monika R. Parikh ${ }^{1}$, Joel B. Winnick ${ }^{1}$, \& Tawnya J. \\ Meadows $^{1}$ \\ ${ }^{1}$ Department of Psychiatry and Behavioral Health, Geisinger \\ ${ }^{2}$ Department of Population Health Sciences, Geisinger
}

\begin{abstract}
Author Note
We have no conflicts to disclose. No grant funding is associated with this work. We thank Ryan Muldowney for co- developing and refining the graphed data of electronic health record metrics included herein. A special thanks to all of our support staff and our primary care colleagues who make our work possible.

Correspondence concerning this article should be addressed to Sean M. O’Dell, MC 4400, 111 N. Academy Ave., Danville, PA, 17822.

Email: sodell@geisinger.edu
\end{abstract}




\begin{abstract}
Objective: In response to the emergence of the COVID-19 pandemic, an initiative to rapidly transition to a telebehavioral health $(\mathrm{TBH})$ service delivery model was developed to maintain access to primary care behavioral health $(\mathrm{PCBH})$ services at Geisinger across a wide, predominantly rural, geographic range. Methods: In line with the "Virtual First" approach employed by the Department of Psychiatry and Behavioral Health, we describe how we prepared for and implemented TBH services while facilitating communication and collaboration among PCBH providers, staff, and trainees, and supported our Geisinger colleagues. Results: We present operational and clinical outcomes of our approach through June 2020, as well as provider surveys of acceptability of the TBH platform used. We found that we were able to attain or surpass comparable patient access and utilization implementing TBH services compared with inperson services over the last two years. We did not observe an increase in proportion of patients with Depression and Anxiety diagnoses during the pandemic. The TBH platform was acceptable overall based on provider ratings. Conclusion: Our Virtual First approach enabled us to maintain access and utilization of PCBH services during a time of widespread uncertainty and vulnerability. We conclude with a discussion of the limitations of the approach used as well as identified next steps in navigating the "new normal" of behavioral healthcare in the age of COVID-19.
\end{abstract}

Keywords: COVID-19, primary care behavioral health, integrated care, telebehavioral health, rural mental health 


\section{Chasing the Curve: Program Description of the Geisinger Primary Care Behavioral Health Virtual First Response to COVID-19}

Beginning in March 2020, health care systems in the United States began to be impacted by the ongoing COVID-19 pandemic, leading to substantial impact on the viability and safety of outpatient services, with downstream implications for health care professionals, patients, and administrators. This increased concerns regarding mental health, substance use, and suicidal ideation given record levels of unemployment, compounding effects of isolation or quarantine, potential trauma related to infection and recovery, and feelings of stress or helplessness during this global pandemic (Torales, O’Higgins, Castraldelli-Maia, \& Ventriglio, 2020). Health care workers are experiencing workplace stressors including pay cuts, layoffs, redeployment, and furloughs. For front line health care workers, scarcity of personal protective equipment, prolonged work hours, fear of infecting family members, and provision of treatment to unprecedented numbers of extremely ill or dying patients may result in traumatic stress events; these stressors can result in fatigue, burn out, feelings of being overwhelmed, and even contracting COVID-19 (Pfefferbaum \& North, 2020). Taken together, access to behavioral health $(\mathrm{BH})$ services quickly emerged as essential in supporting our communities and health systems in the pandemic response.

Moreover, while the destabilization in urban settings resulting early in the COVID-19 pandemic has been documented (Cao et al., 2020), the impact on rural areas may not be fully realized. Prior to the pandemic, rural areas were already typically underserved and access to behavioral health services was limited (Jensen et al., 2020). Clearly, the strain placed upon front line health care workers in already burdened health care systems responding to COVID-19 places individuals living in rural settings with existing disparities in health outcomes at even 
greater risk. Additional burden is placed on families that need to respond to school closings, stay-at-home orders, and physical distancing, masking, and self-isolation requirements that may vary by state or community. With this in mind, existing behavioral health needs for rural communities should be considered along with emerging behavioral health problems in the acute and post-acute phase of the pandemic.

Evidence-informed strategies that can assist individuals during the pandemic in building resiliency, improving communication, enhancing empathic responding, and maintaining positive relationships have been well documented for children (Szabo et al., 2020) and adults (Dan et al., 2020; Weaver et al., 2017); however, given the lack of access to care often experienced in rural settings and social distancing requirements, such as stay-at home orders, a variety of available service delivery modalities are required to meet the current needs of rural communities. Preliminary evidence had been published supporting Telebehavioral health (TBH) as a viable service delivery model for improving patient outcomes, most notably in rural areas where access to care might already be limited (Gonzalez et al., 2015; Hughes et al., 2019). Toward this end, a rapid upscaling of $\mathrm{TBH}$ appeared to be the only viable option to provide necessary behavioral health services during the pandemic. However, health systems face myriad challenges in pivoting to $\mathrm{TBH}$, including information technology infrastructure, regulatory statutes, reimbursement, training, implementation, sustainability, and patient engagement. In addition, even behavioral health providers (BHPs) serving rural areas have expressed hesitancy regarding implementing telehealth due to concerns associated with equivalency with in-person treatment, use of required technology, financial ramifications, and privacy (McClellan et al., 2020).

This article describes activities of the Division of Primary Care Behavioral Health $(\mathrm{PCBH})$ at Geisinger in the rapid development and implementation of $\mathrm{TBH}$ services in response 
to the COVID-19 pandemic from March 2020 through June 2020. We present highlights of the clinical and operational details involved in the response, impact on operational metrics and usability of the TBH platform employed, and then summarize future directions and opportunities to improve $\mathrm{TBH}$ care in the ongoing pandemic response.

\section{Transition to a Virtual First Approach}

PCBH clinics are part of Geisinger Health, a predominantly rural health system located in Central Pennsylvania. Primary care clinicians (PCCs) included physicians, nurse practitioners, and physician assistants. PCCs located in PCBH clinics made in-house referrals to BHPs. Prior to COVID-19, BHP templates consisted of scheduled patient slots and availability for same-day appointment access, warm handoffs (WHOs), and informal "curbside consultations" between PCCs and BHPs. The Department of Psychiatry and Behavioral Health developed a "Virtual First” approach to provide TBH services using the InTouch ${ }^{\circledR}$ platform selected by the Geisinger for use across outpatient service lines, with BHPs working from home or office while patients were seen from their homes. In-person visits were offered only when clinically necessary (e.g., mental health crisis evaluation) at the discretion of the BHP. Demographic characteristics of $\mathrm{PCBH}$ providers are presented in Table 1; a timeline of key operational changes is presented in Figure 1 and is discussed in detail in the following sections.

\section{Preparing for and Implementing Telebehavioral Health Services}

\section{Provider Training in Telebehavioral Health}

TBH services were a new mode of practice for nearly all PCBH team members. Two BHPs elected to complete an online continuing education training in telehealth to advance their practice and benefit their colleagues. The focus on this training was to summarize key considerations for delivering TBH services from home. These BHPs presented a summary of 
lessons learned during a virtual team meeting and developed a summary handout. The PCBH team also posted TBH resources and tip sheets on the Microsoft Teams ${ }^{\circledR}$ page for quick access and review. The PCBH team continued holding regular huddles and monthly team meetings across the COVID-19 transition with time allocated to share tips and tricks, highlight creative clinical approaches, and work through clinical and ethical challenges of TBH.

TBH training and guidelines included a strong focus on documentation requirements and corporate compliance. The PCBH team combined guidance from practice standards, TBH trainings, psychiatry administration, and the telehealth team to identify needed revisions that met all documentation, billing, and TBH requirements. They worked closely with member of the Department's Quality Assurance Team to ensure that standardized documentation in the EHR aligned with broader regulatory requirements.

\section{Communication with Clinic Staff}

Upon initial communication with clinic staff about transition to $\mathrm{TBH}$ services, the $\mathrm{PCBH}$ team worked closely with their respective clinics to ensure that regular communication occurred. BHPs developed a weekly memo for open and frequent communication with PCCs about clinical operation including flexible use of slots to ensure timely access to care, summary of slot availability by BHP, and reminders of our service delivery and availability for WHOs, and crisis evaluations. The memo also provided a resource for providers on stress management and coping and shared patient handouts to use for patient care and education as families are coping in response to COVID-19. BHPs reviewed provider schedules daily, contacted providers about potential WHOs, and communicated a plan for making virtual care 
available instantly to patients. Providers sent a with patient information and coordinated connection to patient device or clinic telephone.

\section{Patient Phone Contacts}

Establishing a platform for phone contact with patients proved challenging. BHPs experienced difficulties keeping their personal cell phone numbers confidential and getting patients to accept phone calls from blocked numbers. The team trialed several platforms to route calls through local clinic numbers and increase patient responsiveness. Cisco Jabber ${ }^{\circledR}$ allowed providers with an individual office number to contact patients directly through that number, but many offices were reached only through group phone lines. Many providers then utilized Google Voice ${ }^{\circledR}$ as a second option. During the rapid transition to use of Google Voice ${ }^{\circledR}$, BHPs did not realize that callers could send text messages to the selected phone number. This platform was quickly abandoned after a patient in crisis sent a text message to a BHP's Google Voice ${ }^{\circledR}$ number overnight. Many providers without individual lines found Doximity ${ }^{\circledR}$ as the best solution as it allowed calls to be sent from a familiar clinic number, prompted patients to return calls through standard hospital channels, and reduced provider liability from missed patient contacts.

\section{Crisis Management}

PCBH leadership developed plans and procedures for high risk patients to decrease likelihood of presenting in the Emergency Department for BH concerns. BHPs continued to ensure that PCCs and clinic staff were aware of the option of a crisis evaluation and/or urgent appointments for high risk patients or patients in crisis. Additionally, a list of county mental health crisis and emergency phone numbers was compiled and made available to all BHPs through the Teams ${ }^{\circledR}$ site to aid in care coordination. 


\section{Staff Redeployment and Maintaining Patient Volumes}

During COVID-19, many PCBH PCCs experienced redeployment, changes in their practice flow, and reduced patient loads due to families not wanting to come into clinic or in efforts to conserve clinic resources. To maintain a full patient schedule for all BHPs and to ensure timely access to BH services, it was necessary for BHPs to broaden their referrals from beyond their de facto $\mathrm{PCBH}$ clinics. Thus, sharing $\mathrm{PCBH}$ patients and incoming referrals became the main redeployment activity of BHPs. TBH services greatly assisted our team's response by redistributing patients from high volume clinics to clinics with open appointments. TBH services also facilitated the redistribution of a BHPs caseload who went out on maternity leave during this time. A weekly report of open slots per clinic for the upcoming four weeks was sent to the PCBH team to guide a plan for sharing new patients across clinic and to crisis support services. The adult PCBH team paired two clinics to share patient caseload, while the pediatric PCBH team distributed patients broadly across the entire team.

\section{Facilitating Communication and Collaboration Among PCBH Providers and Staff}

\section{File Sharing}

The PCBH team utilized Microsoft Teams ${ }^{\circledR}$ to securely share materials and resources due to ease of access from home office settings and the ability for multiple team members to edit files and pages simultaneously. One BHP was identified to lead structuring, organizing, and managing incoming materials during work from home. The resultant page included materials in the following categories: COVID-19 patient resources, COVID-19 provider resources, meeting and huddle agendas, patient handouts used in typical clinical practice, a record of communications from division of psychiatry and hospital leadership, telehealth procedures and 
fast facts, and technology guidance and support materials. This page allowed providers to efficiently locate materials needed for clinical work and administration from their home offices.

\section{Coordinating Procedures and Communication}

Early after the transition to TBH services, BHPs primarily used emails for questions and problem-solving. Email volume quickly became burdensome and tracking of procedures was challenging. In response, PCBH leadership created spreadsheet on Teams ${ }^{\circledR}$ for collection, response, and tracking of procedural details. PCBH leadership and telehealth leadership reviewed this document frequently to review accuracy of responses to evolving procedures.

At the start of TBH services, all scheduled patients needed to receive a phone contact prior to their first TBH visit. BHPs contacted each of their scheduled patients by phone to inform them of the transition to $\mathrm{TBH}$ services and confirm contact information. In standard procedures, appointment changes were made via individual phone messages. To improve scheduling efficiency, PCBH leadership team collaborated with the support staff team and created password-protected files for tracking needed appointment actions across all BHPs. Password protected files were also used to track clinical groups of patients including high risk patients needing care in the case of provider absence in accordance with HIPAA regulations.

\section{Supervision for PCBH trainees}

PCBH supervisors were regularly available by phone, secure instant message, and other forms of virtual contact to ensure support of PCBH trainees. If a supervisor was out of the office, all PCBH supervisors were available for back-up coverage. The PCBH team maintained two hours of scheduled supervision weekly and incorporated clinic huddles to ensure clear communication. Supervisors incorporated discussions about maintaining work-life balance and the complexity of delivering TBH services at home. To meet requirements for direct 
observations of patient care, supervisors coordinated with trainees on remotely connecting to the patient's TBH appointment.

\section{Supporting Geisinger Colleagues and PCBH Trainees}

Geisinger's Center for Professionalism and Well-Being developed a program titled, Project RISE (Resilience In Stressful Events), which includes a 3-tiered model to help mitigate the adverse behavioral health effects on healthcare providers during COVID-19. A call for volunteers was sent to providers across the health system and 11 BHPs volunteered to provide peer support services for providers in need or that are identified to need additional support (e.g., one-on-one crisis intervention). Project RISE volunteers completed a one-hour online training and submitted their availability to provide this service throughout the week.

\section{Operational and Clinical Outcomes of Virtual First Approach}

We used indicators from the Geisinger Clinical Data Intelligence System (i.e., data warehouse of electronic health record data) on operational and clinical characteristics of behavioral healthcare delivered within PCBH clinics from January 2018 until June 2020 to compare our Virtual First approach to TBH during the COVID-19 pandemic thus far to previous years. We examined several outcomes of interest, including show rates, percentage of new patients, whether these varied with patient distance from clinic. Trends in these domains were consistent with past years. Next, we describe the most salient trends we observed.

\section{Behavioral Healthcare Utilization}

Completed encounters and the percentage of patients seen for an initial appointment within 10 days were graphed for Adult and Pediatric PCBH clinics (Figure 3). These metrics are considered opposing because as patient volume increases, the available appointments in the next 
10 days naturally decreases and so achieving success on both metrics simultaneously is challenging. This trend is apparent for both groups, with Pediatric PCBH crossing this threshold in October 2018 at around 1,300 completed encounters per month and Adult PCBH in March 2019 around 800 completed encounters per month. Adult PCBH was closing the gap on this trend throughout Winter 2019 and was able to maintain time to seen metrics comparable to March 2019 after the transition to Virtual First care while increasing completed encounters to unprecedented levels. The Pediatric PCBH group began to close the time to seen gap throughout the Virtual First period to the highest levels within the observed period while maintaining comparable completed encounter volume to Spring 2019.

\section{Care for Patients with Anxiety and Depression}

The scientific literature foreshadowed the possibility of increases in internalizing problems associated with self-quarantine, stay-at-home orders, loss of employment, school closings, and other disruptions to daily routines during the first wave of COVID-19 in the US (e.g., Pfefferbaum \& North, 2020). As such, we were interested in examining any changes in the proportion of patients with anxiety and depression treated throughout the Virtual First response to COVID-19. We examined the percentage of patients with ICD-10 codes for Anxiety (ICD-10 F40-F48) and Depression (ICD-10 F32-F39) as billing diagnoses for completed encounters within the project period were graphed to determine if any change in level or slope were evident.

For Adult $\mathrm{PCBH}$, no discernable trend was evident based on age segments, so data from all participants were combined into one trend line (Figure 4). Over the last two years, there was an increasing trend in Anxiety prevalence treated within Adult PCBH. Anxiety diagnoses peaked at $41 \%$ of all patients with a completed encounter in April 2020, an increase of $6 \%$ compared with April 2019. Depression prevalence in 2018 arched throughout the year before beginning to 
rise again in February 2019. This trend plateaued in June 2019 and maintained between 43\% and $47 \%$ of patients with competed encounters through June 2020.

Pediatric PCBH evidenced clear trends according to age segment for Anxiety and Depression as depicted in Figure 4, with higher overall proportions of youth with Anxiety and Depression diagnoses for older age segments. The only age segment with increasing trends during the first wave of COVID-19 for Anxiety were youth aged 13-18; however, prevalence of youth treated for Anxiety disorders appears consistent with trends occurring over the last two years. Youth aged 13-18 treated for Depression did increase overall compared to previous years and the increasing proportion throughout March through June 2020 contrasts with falling proportions over the same months in 2018 and 2019. Taken together, there is indication that Depression was a more prevalent concern throughout the first wave of COVID-19 within pediatric patients served in $\mathrm{PCBH}$.

\section{Usability of Telebehavioral Health Platform}

Views of BHPs using the InTouch ${ }^{\circledR}$ Home platform to deliver behavioral healthcare during the Virtual First initiative was measured during the month of June 2020 using the Telehealth Usability Questionnaire (TUQ; Parmanto, Lewis, Graham, \& Bertolet., 2016). Table 2 displays ratings across the six factors of the TUQ. Overall, ratings showed moderate to high acceptability across domains, with the Reliability factor receiving the lowest ratings for both Adult and Pediatric PCBH providers. There were no statistically significant differences between groups on average ratings.

\section{Limitations}


Although there are strengths in the Geisinger PCBH approach to rapid uptake of TBH services during the first months of the COVID-19 pandemic, limitations to our approach should also be considered. Because our primary focus was on maintaining clinical operations through $\mathrm{TBH}$ in the midst of a pandemic, we have relatively limited clinical data on outcomes of our Virtual First approach. Reliance on billing diagnoses instead of prospective screening for internalizing concerns, for instance, has important limitations when characterizing patient segments and tracking the effectiveness of our clinical response. This approach also cannot detect other important patient segments for whom billing diagnoses do not exist or would not typically be billed in outpatient psychotherapy appointments. For instance, Shi and colleagues (2020) reported on a population-based survey conducted in China and found that those most likely to have negative mental health outcomes were those who were in self-quarantine or had delays in returning to work, and that rates of insomnia and acute stress were high. Prospective and proactive screening is needed to overcome these limitations as the $\mathrm{PCBH}$ response to the pandemic progresses. Related to this, the InTouch ${ }^{\circledR}$ platform used during the TBH roll out was selected based on what could be implemented across the entire health system to shift to virtual care as quickly as possible. This tradeoff came with the cost of not being able to provide psychotherapy groups or shared office visits on the platform, which limited our ability to organize preventive and targeted services to reach patients most efficiently and it appears likely that access to beneficial services was limited as a result. Finally, we are also limited in the conclusions we can draw about our Virtual First approach by the absence of systematic feedback from our primary care colleagues and the families we serve. Without such feedback, it is not clear the extent to which the services we are offering are meeting the needs of these important stakeholder groups. 


\section{Future Directions}

Consistent with the mission of Geisinger PCBH to improve access to high-quality behavioral health care, we navigated the complexity of a rapid transition to TBH services in response to the COVID-19 pandemic using a Virtual First approach and demonstrated commendable patient engagement and teamwork. Having navigated the first wave of the pandemic, the next steps in our response involve actively shaping the "new normal" of behavioral healthcare and striving for comprehensive care that is sensitive and responsive to the needs of patients we serve and the colleagues we partner with in PCBH. It does not appear that the alternative to TBH services will resemble in-person care delivered before the pandemic any time soon, as the infection control procedures in clinics now involve masking, physical distancing, and in some cases physical barriers. And so, it behooves us to embrace TBH as a modality that can safely increase the reach and effectiveness of our services.

Because integrated care has been described as a process of building the plane while flying it (Khatri, 2014), it may not be surprising that we were able to find some success and collaboratively embrace the sweeping and rapid changes involved in the Virtual First approach while contributing to Project RISE and maintaining a training program. In keeping with this metaphor, it seems important to focus on continuous improvement of the instrument panel on the plane to help us better navigate the uncharted skies we find ourselves in. The development of scalable data-based decision making in health care writ large has been slow-going, likely owing to the complexity in aligning the scientific, informatics, financial, and cultural priorities needed to enable a "learning" health care system (Institute of Medicine, 2013). Embracing this approach in behavioral health now could have major advantages in navigating the pandemic response, including better access to actionable data to improve the care experience, engaging patients in 
care and self-management, and partnering effectively with patient, provider, and community stakeholders to address barriers to care (Stein et al., 2016).

Shaping the new normal of behavioral health care in the age of COVID-19 must also involve actively working to leave behind unworkable vestiges of legacy service delivery models. It is more apparent than ever that a fee-for-service reimbursement model aligned with "sick care" for fully manifested psychiatric disorders is a major constraining factor to integration and is incompatible with a learning behavioral health care system. Capitated models with incentives for prevention and health promotion through collaboration between clinicians, transdisciplinary researchers, patients, and other relevant stakeholders to work for the benefit of vulnerable patient segments are urgently needed in its place (Holmes et al., 2020).

Promotion of strategies that facilitate openness and awareness to engage in meaningful activities even with the challenges brought on by the pandemic are also needed to address the known repertoire narrowing effects of anxiety, fear, and self-isolation that are predictable consequences of awareness of ongoing events in the pandemic and adherence to essential behavioral guidelines to mitigate the spread of SARS-CoV-2 (Presti, McHugh, Gloster, Karkela, \& Hayes, 2020). Practical skills for effectively coping with the pandemic exist (e.g., Harris, 2020) and can be disseminated widely and reinforced in primary care (Dalton, Rapa, \& Stein, 2020). Actionable educational measures are important, as demonstrated by emerging evidence in an adolescent sample showing that mindsets on seriousness of the pandemic, social responsibility, social trust, and self-interest predicted adherence to behavioral guidelines to reduce spread of COVID-19 and resource hoarding behavior (Oosterhoff \& Palmer, 2020). This work also needs to be specifically tailored to identifying and responding to the unique needs of individuals living in rural communities, as research demonstrates these do not always align with 
those in suburban and urban communities in intuitive ways (e.g., Tolliver, Polaha, Williams, \& Studts, 2020).

\section{Conclusion}

These times represent a defining moment for pediatric primary care telehealth (Fiks, Jenssen, \& Ray, 2020). Like the temporary relaxation of regulations on provision of TBH services that enabled the Virtual First approach described herein, it remains unclear how long the window of opportunity will remain open enact meaningful and lasting change in PCBH services. Diligent, planful, and coordinated efforts are needed as we enter into the next phase of implementation in Geisinger $\mathrm{PCBH}$ to rise to the challenges faced in the ongoing response to the COVID-19 pandemic. 


\section{References}

Adalja, A. A., Toner, E., \& Inglesby, T. V. (2020). Priorities for the US Health Community Responding to COVID-19. JAMA - Journal of the American Medical Association, 2019, 2019-2020. https://doi.org/10.1001/jama.2020.3413

Allen, T., Grier-Reed, T., \& Maples, A. (2020). On the frontier: Exploring rural psychologist practice in integrated behavioral health care. Journal of Rural Mental Health, 44(2), 106117. https://doi.org/10.1037/rmh0000132

Armitage, R., \& Nellums, L. B. (2020). Considering inequalities in the school closure response to COVID-19. The Lancet Global Health, (20), 30116. https://doi.org/10.1016/s2214$\underline{109 x(20) 30116-9}$

Bai, Y. (2020). Presumed Asymptomatic Carrier Transmission of COVID-19. The New England Journal of Medicine, 382(13), 1199-1207. https://doi.org/10.1056/NEJMoa2001316

Biglan, A., Elfner, K., Garbacz, S. A., Komro, K., Prinz, R. J., Weist, M. D., ... Zarling, A. (2020). A Strategic Plan for Strengthening America's Families : A Brief from the Coalition of Behavioral Science Organizations. Clinical Child and Family Psychology Review, 23(2), 153-175. https://doi.org/10.1007/s10567-020-00318-0

Cao, W., Fang, Z., Hou, G., Han, M., Xu, X., Dong, J., \& Zheng, J. (2020). The psychological impact of the COVID-19 epidemic on college students in China. Psychiatry Research, 112934. https://doi.org/10.1016/j.psychres.2020.112934

Castagnoli, R., Votto, M., Licari, A., Brambilla, I., Bruno, R., Perlini, S., ... Marseglia, G. L. (2020). Severe Acute Respiratory Syndrome Coronavirus 2 (SARS-CoV-2) Infection in 
Children and Adolescents: A Systematic Review. JAMA Pediatrics, 2.

https://doi.org/10.1001/jamapediatrics.2020.1467

Dalton, L., Rapa, E., \& Stein, A. (2020). Protecting the psychological health of children through effective communication about COVID-19. The Lancet Child \& Adolescent Health, 4642(20), 19-20. https://doi.org/10.1016/S2352-4642(20)30097-3

Dan, D. K., Herschell, A. D., Bodea-Crisan, T., Schake, P. L., \& Gavin, J. G. (2020). The Core 4 Clinical Model: Strengthening the rural behavioral health workforce through a focus on foundational clinical skills. Journal of Rural Mental Health, 44(2), 118-128. https://doi.org/10.1037/rmh0000133

Dong, Y., Mo, X., Hu, Y., Qi, X., Jiang, F., Jiang, Z., \& Tong, S. (2020). Epidemiological Characteristics of 2143 Pediatric Patients With 2019 Coronavirus Disease in China. Pediatrics. https://doi.org/10.1542/peds.2020-0702

Gonzalez, G. E., \& Brossart, D. F. (2015). Telehealth videoconferencing psychotherapy in rural primary care. Journal of Rural Mental Health, 39(3-4), 137-152. https://doi.org/10.1037/rmh0000037

Hagler, M., Grych, J., Banyard, V., \& Hamby, S. (2016). The ups and downs of self-regulation: Tracing the patterns of regulatory abilities from adolescence to middle adulthood in a rural sample. Journal of Rural Mental Health, 40(3-4), 164-179. https://doi.org/10.1037/rmh0000053

Harris, R. (2020). FACE COVID: How to respond effectively to the Corona crisis. Retreived from https://www.actmindfully.com.au/wp-content/uploads/2020/03/FACE-COVIDeBook-by-Russ-Harris-March-2020.pdf, on July 19, 2020. 
Hughes, M. C., Gorman, J. M., Ren, Y., Khalid, S., \& Clayton, C. (2019). Increasing access to rural mental health care using hybrid care that includes telepsychiatry. Journal of Rural Mental Health, 43(1), 30-37. https://doi.org/10.1037/rmh0000110

Institutes of Medicine (2013). Best care at lower cost: the path to continuously learning health care in America. Edited by M. Smith, R. Saunders, L. Stuckhardt, et al. Washington, DC, National Academies Press, Institute of Medicine.

Jensen, E. J., Wieling, E., \& Mendenhall, T. (2020). A phenomenological study of clinicians' perspectives on barriers to rural mental health care. Journal of Rural Mental Health, 44(1), 51-61. https://doi.org/10.1037/rmh0000125

Kerr, M. M., Fried, S. E., Price, R. H., Cornick, C., \& Dugan, S. E. (2017). Rural children's responses to the Flight 93 crash on September 11, 2001. Journal of Rural Mental Health, 41(3), 176-188. https://doi.org/10.1037/rmh0000072

Khatri, P. (2014). CFHA's past, present, and future: Building the plane while flying. Families, Systems, \& Health, 32(4), 446-447. https://doi.org/10.1037/fsh0000091

Kim, S.-W., \& Su, K.-P. (2020). Using psychoneuroimmunity against COVID-19. Brain, Behavior, and Immunity, (2), 0-1. https://doi.org/10.1016/j.bbi.2020.03.025

Komaromy, M., Madden, E. F., Hager, B., Qeadan, F., Ceballos, V., Duhigg, D., ... Arora, S. (2019). Improvement in behavioral health symptoms and functioning among rural patients cared for by primary care teams using the extension for community health care outcomes model. Journal of Rural Mental Health, 43(2-3), 73-80.

https://doi.org/10.1037/rmh0000115 
Liu, J. J., Bao, Y., Huang, X., Shi, J., \& Lu, L. (2020). Mental health considerations for children quarantined because of COVID-19. The Lancet Child \& Adolescent Health, 2019(20), 2019-2020. https://doi.org/10.1016/s2352-4642(20)30096-1

Lu, X., Zhang, L., Du, H., Zhang, J., Li, Y. Y., Qu, J., ... Wong, G. W. K. (2020). SARS-CoV-2 Infection in Children. New England Journal of Medicine, 1-3. https://doi.org/10.1056/NEJMc2005073

McClellan, M. J., Florell, D., Palmer, J., \& Kidder, C. (2020). Clinician telehealth attitudes in a rural community mental health center setting. Journal of Rural Mental Health, 44(1), 6273. https://doi.org/10.1037/rmh0000127

Pfefferbaum, B., \& North, C. S. (2020). Mental health and the Covid-19 pandemic. New England Journal of Medicine, 1-3. https://doi.org/DOI: 10.1056/NEJMp2008017

Smith, A. J. (2018). Determinants of Transgender Individuals' Well-Being, Mental Health, and Suicidality in a Rural State. Journal of Rural Mental Health, 42(2), 116-132. https://doi.org/10.1016/j.physbeh.2017.03.040

Szabo, T. G., Richling, S. M., Embry, D. D., Biglan, A., \& Wilson, K. G. (2020). From Helpless to Hero : Promoting Values-Based Behavior and Positive Family Interaction in the Midst of COVID-19. Behavior Analysis in Practice. https://doi.org/10.1007/s40617-020-00431-0

Tolliver, M., Polaha, J., Williams, S. L., \& Studts, C. R. (2020). Evaluating the prevalence of child psychosocial concerns in rural primary care. Journal of Rural Mental Health, 44(2), 96-105. https://doi.org/10.1037/rmh0000130 
Torales, J., O’Higgins, M., Castaldelli-Maia, J. M., \& Ventriglio, A. (2020). The outbreak of COVID-19 coronavirus and its impact on global mental health. International Journal of Social Psychiatry, 66(4), 317-320. https://doi.org/10.1177/0020764020915212

Weaver, A., \& Himle, J. A. (2017). Cognitive--behavioral therapy for depression and anxiety disorders in rural settings: A review of the literature. Journal of Rural Mental Health, 41(3), 189-221. https://doi.org/10.1037/rmh0000075 
Table 1

Demographic Characteristics of Primary Care Behavioral Health Providers

\begin{tabular}{|c|c|c|c|c|}
\hline \multirow[t]{2}{*}{ Characteristic } & \multicolumn{2}{|c|}{ Adult } & \multicolumn{2}{|c|}{ Pediatric } \\
\hline & $n$ & $\%$ & $n$ & $\%$ \\
\hline Female & 8 & 100 & 18 & 77.8 \\
\hline \multicolumn{5}{|l|}{ Race } \\
\hline White or Caucasian & 5 & 75.0 & 16 & 878.9 \\
\hline Asian or Asian American & 2 & 0 & 1 & 5.6 \\
\hline Mixed Race & 0 & 25.0 & 1 & 5.6 \\
\hline Hispanic/Latinx & 0 & 0 & 1 & 5.6 \\
\hline \multicolumn{5}{|l|}{ Discipline } \\
\hline Psychology & 6 & 75.0 & 15 & 83.3 \\
\hline Social Work & 2 & 25.0 & 1 & 5.6 \\
\hline $\begin{array}{l}\text { Licensed Professional } \\
\text { Counselor }\end{array}$ & 0 & 0 & 2 & 11.1 \\
\hline Trainee & 0 & 0 & 7 & 38.9 \\
\hline Licensed & 6 & 100 & 11 & 61.1 \\
\hline
\end{tabular}

Note. $N=26$ ( $n=8$ for Adult PCBH and $n=18$ for Pediatric PCBH). Participants were on average 35.6 years old $(S D=3.29)$ with 2.6 years in current position $(S D=1.66)$ in the Adult PCBH group, and 33.4 years old $(S D=6.01)$ with 3.1 years in current position $(S D=2.78)$ in the Pediatric PCBH Group. 
Table 2

Primary Care Behavioral Health Provider Ratings of the InTouch Home Telemedicine Platform on the Telehealth Usability Questionnaire

\begin{tabular}{lcccc}
\hline Factor & \multicolumn{2}{c}{ Adult } & \multicolumn{2}{c}{ Pediatric } \\
\cline { 2 - 5 } & $M$ & $S D$ & 6.22 & 0.75 \\
\hline Usefulness & 5.96 & 0.95 & 5.63 & 0.68 \\
Ease of Use \& Learnability & 6.21 & 0.59 & 4.76 & 0.91 \\
Interface Quality & 5.09 & 0.61 & 4.50 & 1.10 \\
Interaction Quality & 4.94 & 1.14 & 3.50 & 1.11 \\
Reliability & 4.25 & 1.00 & 5.88 & 0.85 \\
Satisfaction \& Future Use & 5.86 & 0.87 & & \\
\hline
\end{tabular}

Note. Anchors: 1-Disagree, 7-Agree 


\section{Figure 1}

Location of Adult and Pediatric Primary Care Behavioral Health Clinics

\section{Adult PCBH Clinics}

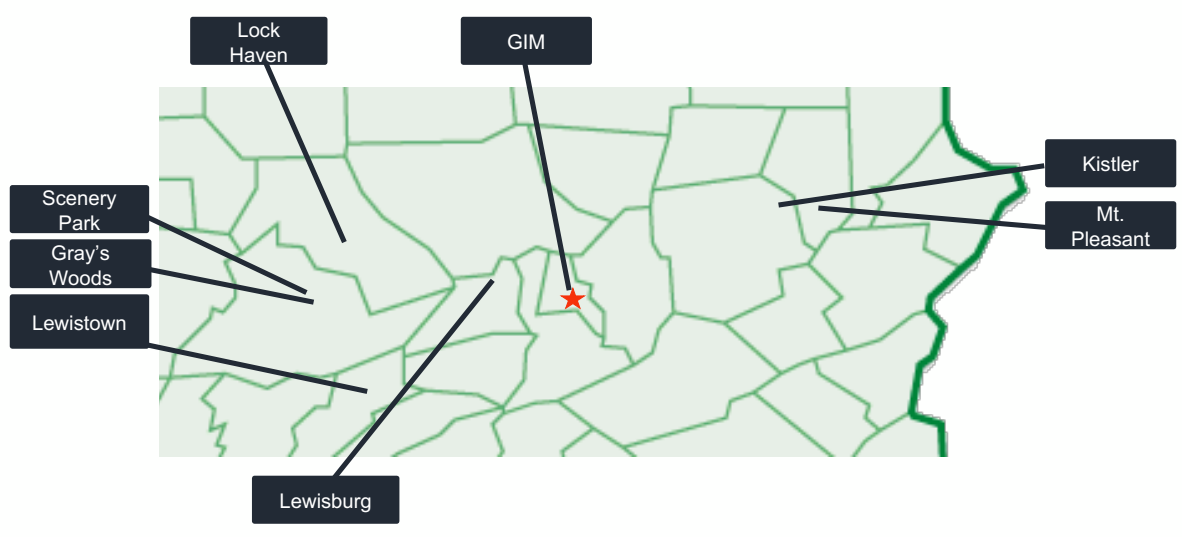

\section{Pediatric PCBH Clinics}

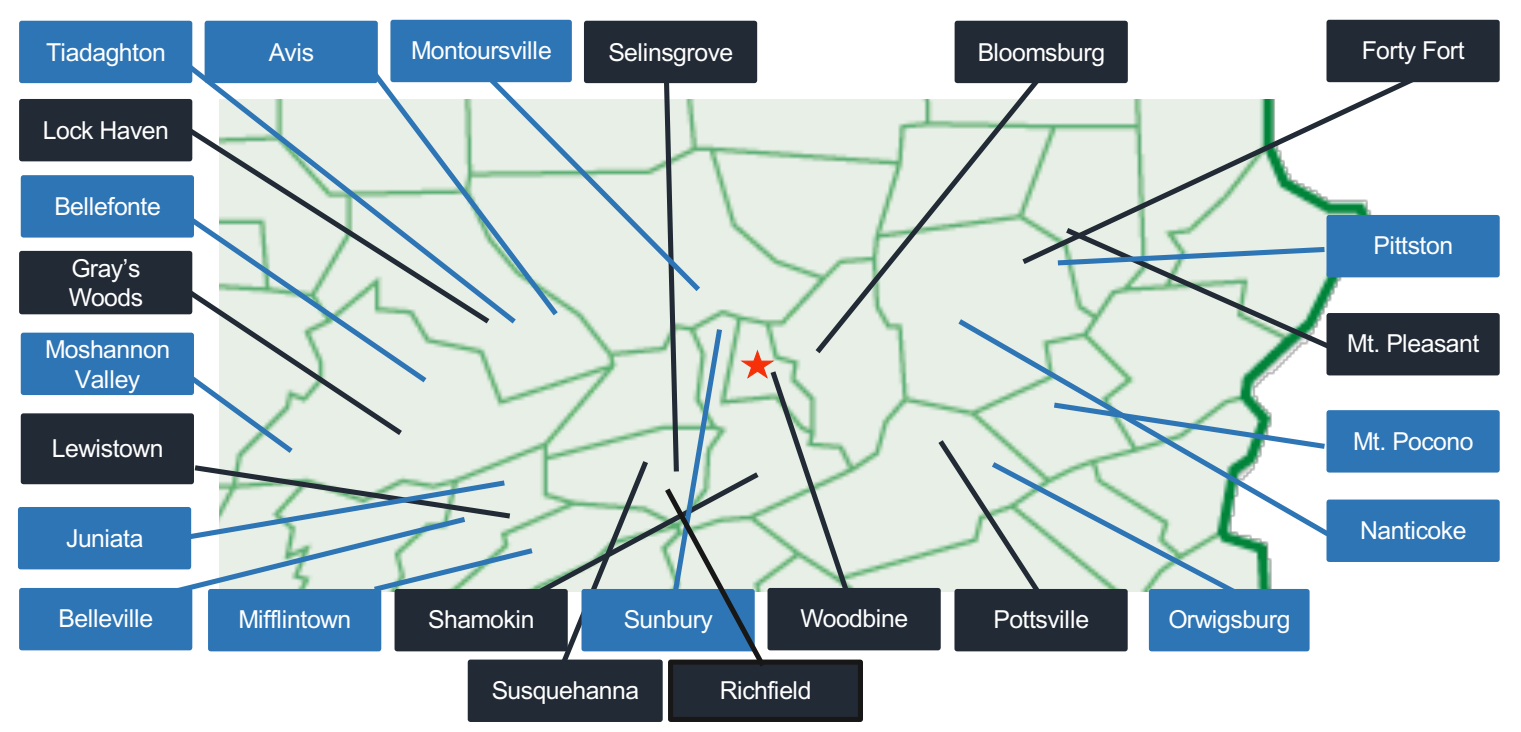

Note. GIM, Geisinger Internal Medicine. Location of each PCBH clinic is shown on a

Pennsylvania county map; the red star indicates the location of the main hospital campus in

Danville, PA. Dark blue boxes indicate clinics staffed with a co-located behavioral health 
provider (i.e., hub clinics) and light blue boxes indicate pediatric PCBH clinics that send behavioral health referrals for their patients to a behavioral health provider at a hub clinic (i.e., extension clinics). 
Figure 2

Timeline of Transition to Virtual First Telebehavioral Health Services within Geisinger Primary Care Behavioral Health Clinics

\section{Friday, 3/13/20}

-Governor Wolfe announced statewide school closings for Pennsylvania

-Psychiatry leadership contacted telehealth leadership team to

begin conversations about

converting services

\section{Monday, 3/16/20 - Friday, 3/20/20}

-Telehealth team provided virtual trainings at $7 \mathrm{am}$,

Noon, and $5 \mathrm{pm}$ to prevent conflict with clinical

schedules. All PCBH staff completed this training

-PCBH staff completed online quality assurance course

and submitted privileging form to request emergency

telemedicine privileges

-BHPs started piloting telehealth platform

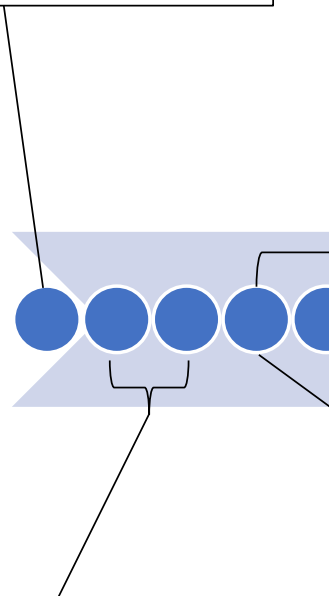

Saturday $3 / 14 / 20$ - Sunday,

\section{$3 / 15 / 20$}

-PCBH leadership submitted

information on staffing, clinic

locations and addresses, staffing

priorities, and equipment needs

to telehealth administration

converting services

$$
\begin{aligned}
& \text { Monday, } \mathbf{3 / 1 6 / 2 0} \\
& \text {-Telehealth team sent equipment } \\
& \text { to BHPs via interoffice mail } \\
& \text { system } \\
& \text {-PCBH administration submitted } \\
& \text { additional information to assist in } \\
& \text { telehealth platform set up } \\
& \text { including provider names, EHR } \\
& \text { scheduling instructions }
\end{aligned}
$$

Note. Timeline of key events between May 13, 2020 and April 10, 2020 involved in the transition to telebehavioral health services

during the COVID-19 pandemic within Geisinger Primary Care Behavioral Health clinics. 


\section{Figure 3}

Adult and Pediatric Primary Care Behavioral Health Percentage of Patients Seen in Ten Days and Number of Completed Encounters January 2018 to June 2020

\section{Adult PCBH}

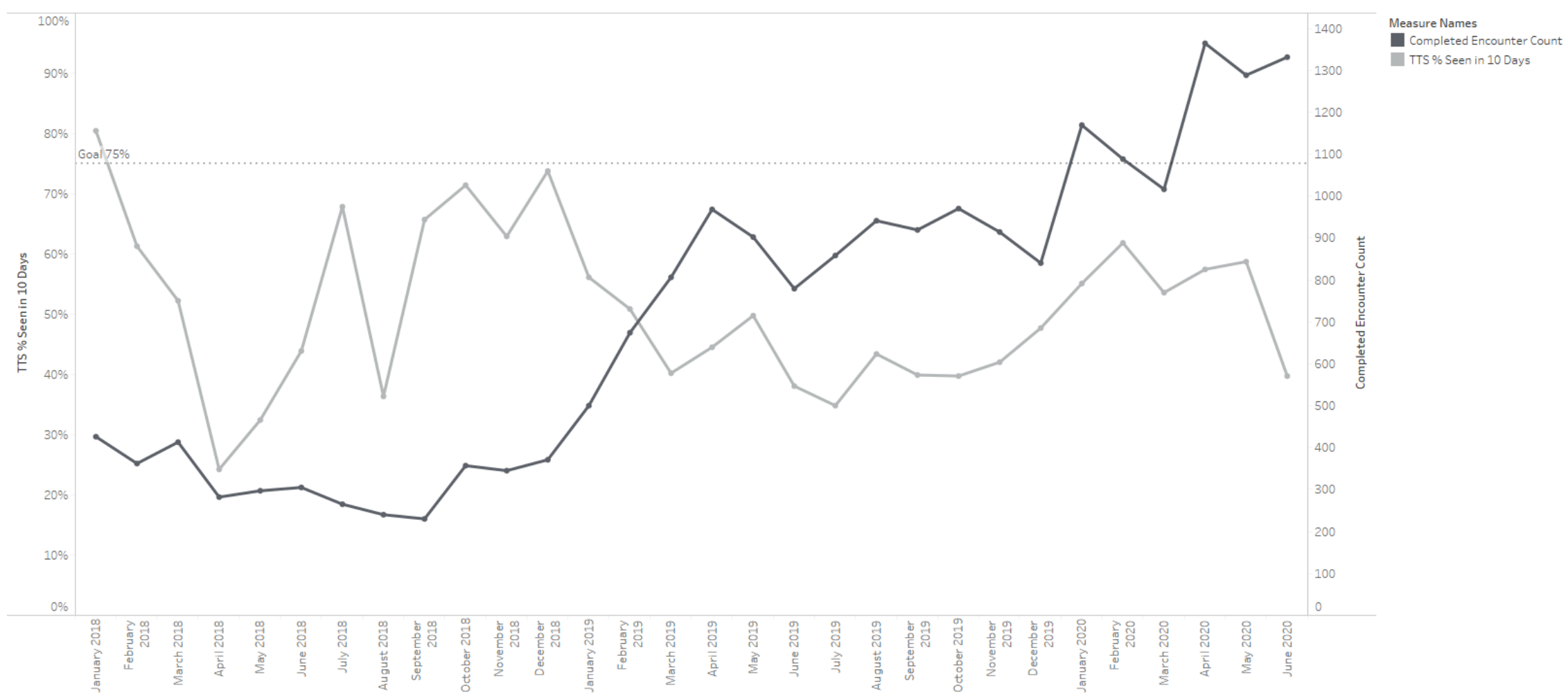




\section{Pediatric PCBH}

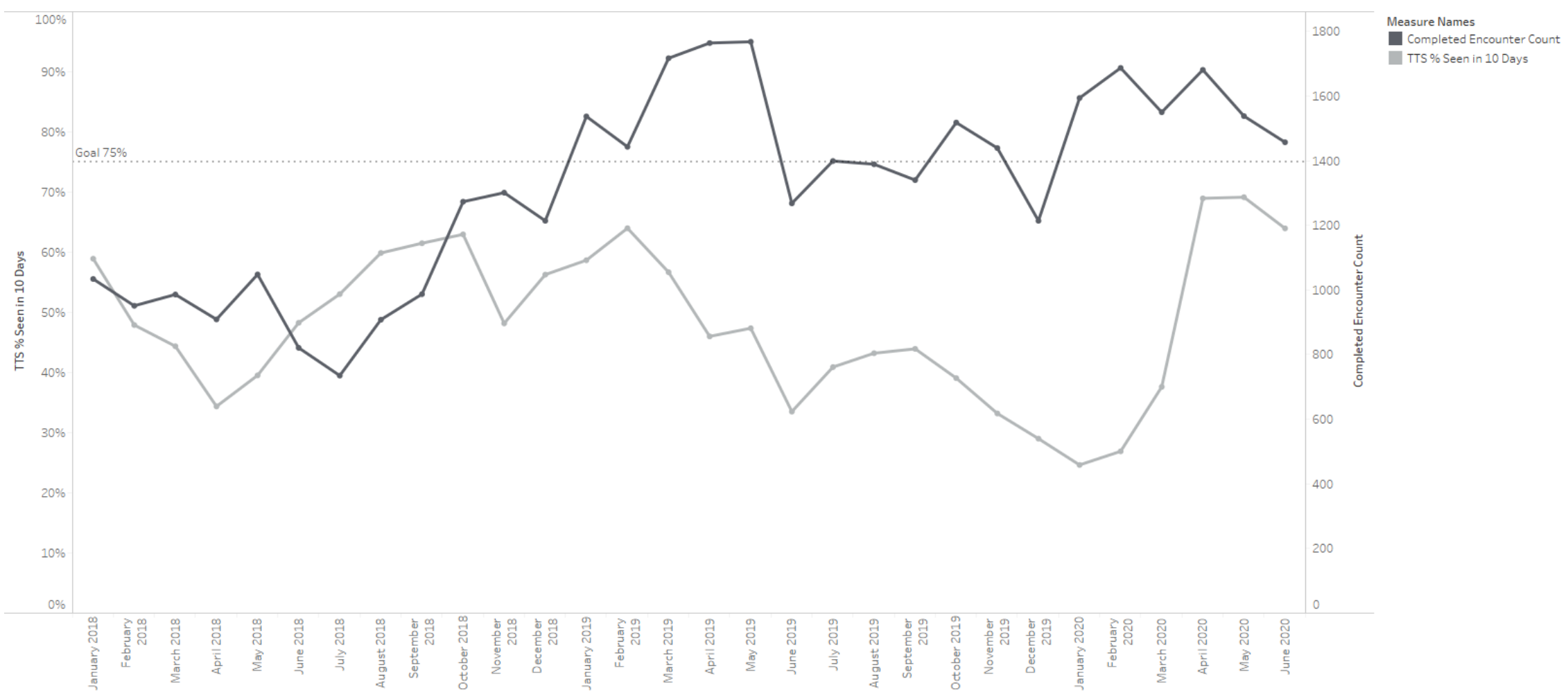

Note. The percentage of patients with a latency of ten or fewer days between the time the appointment was made and the first appointment is shown on the left y-axis. The dashed line represents the Health System goal of $75 \%$ of patients seen within 10 days.

The Right y-axis depicts the number of completed encounters. 


\section{Figure 4}

Adult and Pediatric Primary Care Behavioral Health Percentage of Patients Seen with Anxiety and Depression January 2018 to June 2020

Adult PCBH

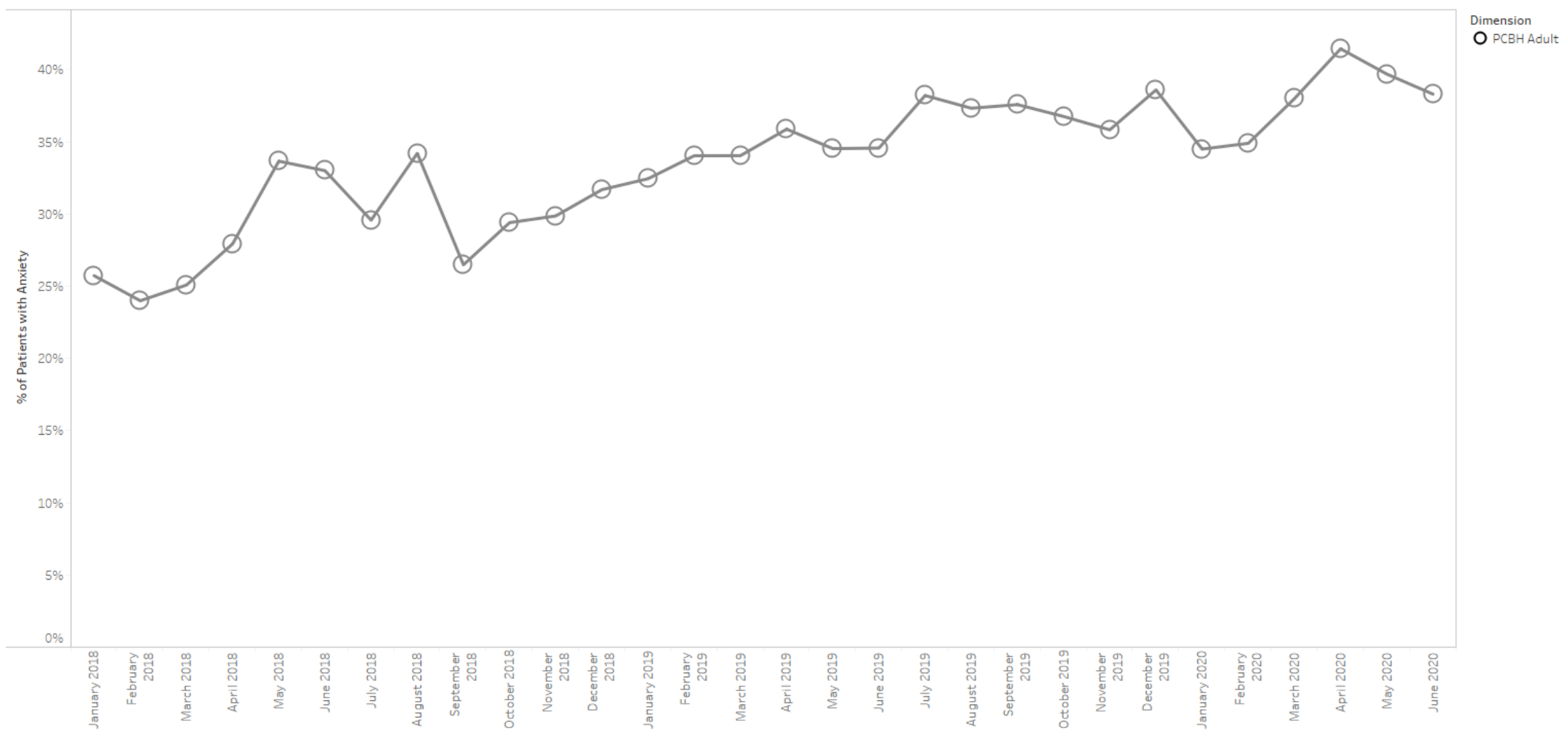




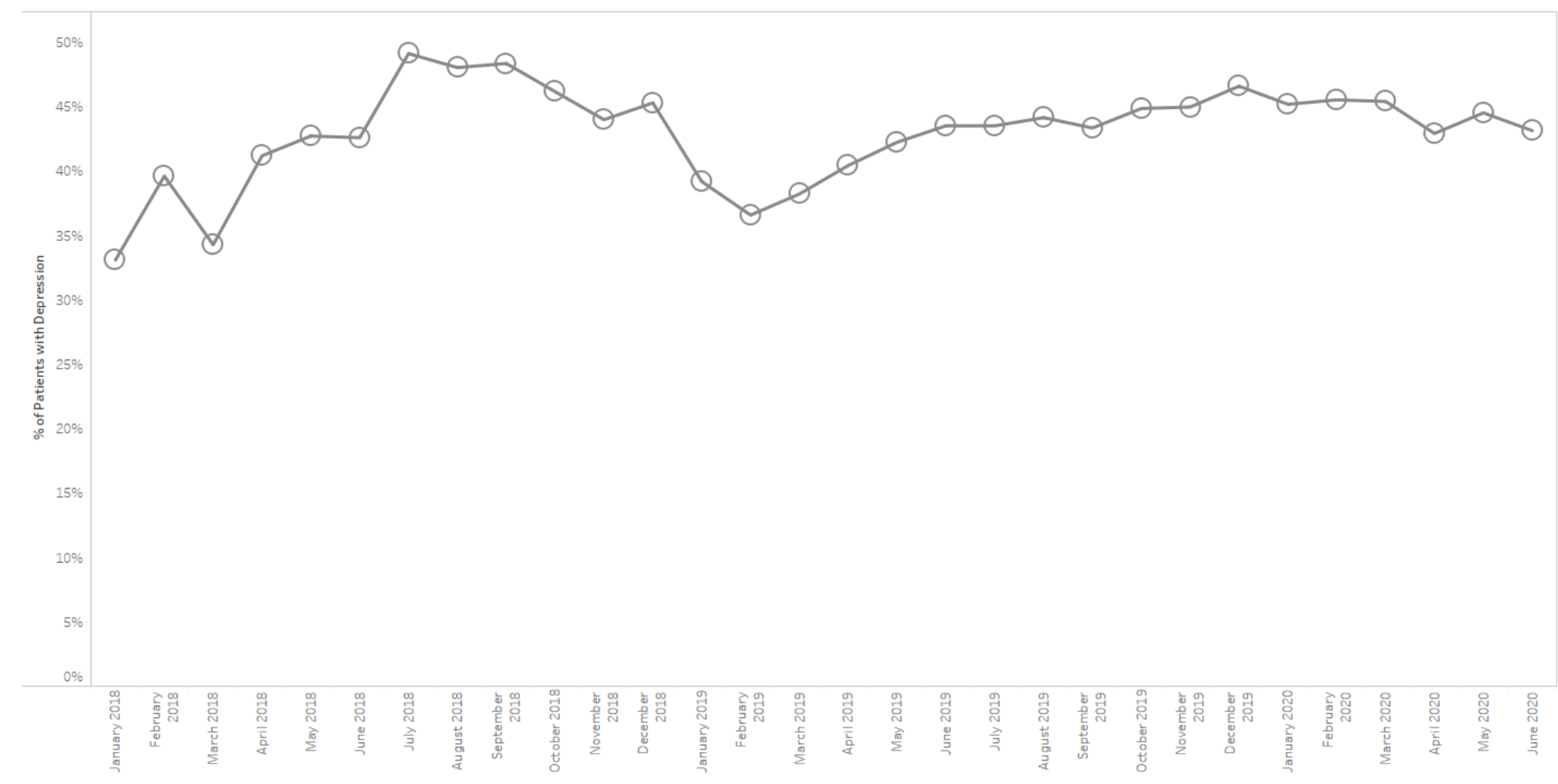

Dimension

P PCBHAdult 


\section{Pediatric PCBH}

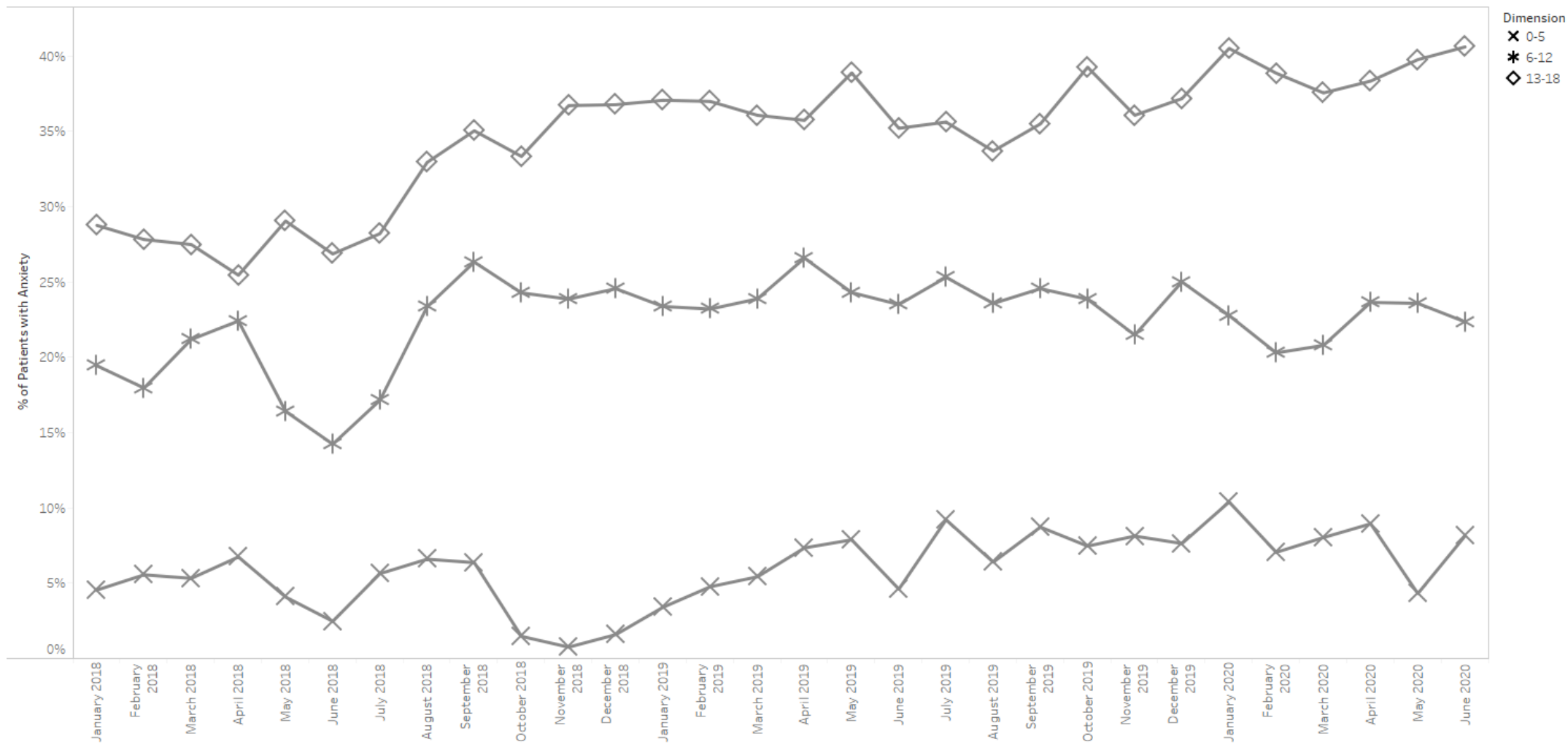




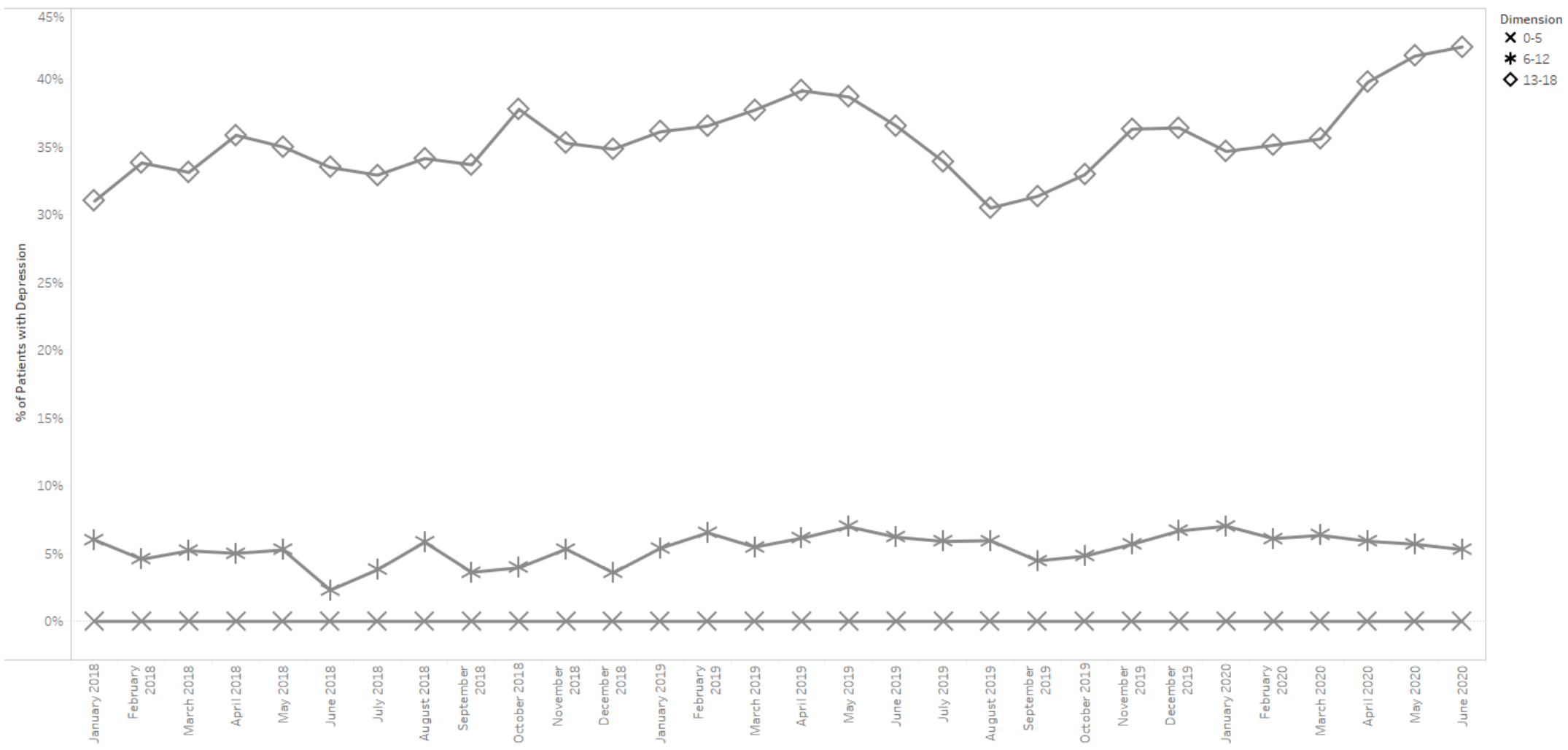

Note. The percentage of patients with a billing diagnosis categorized as Anxiety or Depression were graphed monthly from January 2018 through June 2020. Because Adult PCBH percentages did not yield trends that varied by age segments, these data are graphed for all patients represented by a single line on the graphs. Pediatric PCBH percentages of patients with and Anxiety and Depression disorder diagnosis are graphed across 0-5, 6-10, and 13-18 age segments. 